is of course the multiplication in $A_{1}$ ). By the proof of Theorem 2 , in order to show the equivalence of $A$ and $A_{1}$ it is sufficient to show that $[w, w]=\gamma f$ and $[w, z]=[z U, w]$ for every $z$ of $R$. But $[w, w]$ $=w\left(f^{-1} w\right)=(f g)\left(f^{-1} f g\right)=f g^{2}=\gamma f$, and $[w, z]=w\left(f^{-1} z\right)=(f g)\left(f^{-1} f x\right)$ $=(f g) x=g(x \cdot f S)=(f \cdot x S) g=(f \cdot x S)\left(f^{-1} f g\right)=z U\left(f^{-1} w\right)=[z U, w]$. This proves the theorem.

Newport News, Va.

\title{
ON FIBRE SPACES. I
}

RALPH H. FOX

In subsequent papers I propose to investigate various properties of fibre spaces. ${ }^{1}$ The object of the fundamental Hurewicz-Steenrod definition ${ }^{1}$ is to state a minimum ${ }^{2}$ set of readily verifiable conditions under which the covering homotopy theorem ${ }^{1}$ holds. An apparent defect of their definition is that it is not topologically invariant. In fact, for topological space $X$ and metrizable non-compact space $B$ the property " $X$ is a fibre space over $B$ " depends on the metric of $B$. The object of this note is to give a topologically invariant definition of fibre space and to show that (when $B$ is metrizable) $X$ is a fibre space over $B$ in this sense if and only if $B$ has a metric in which $X$ is a fibre space over $B$ in the sense of Hurewicz-Steenrod. Since the definition of fibre space is controlled by the covering homotopy theorem, an essential part of my program is to give a topologically invariant definition of uniform homotopy.

Let $\pi$ be a continuous mapping of a topological space $X$ into another topological space $B$. Let $\Delta=\Delta(B)$ denote the diagonal set $\sum_{b \in B}(b, b)$ of the product space $B \times B$ and let $\bar{\pi}$ denote the mapping of $X \times B$ into $B \times B$ which is induced by the mapping $\pi$ according to the rule $\bar{\pi}(x, b)=(\pi(x), b)$. Thus the graph $G$ of $\pi$ is the set $\bar{\pi}^{-1}(\Delta)$, and $\bar{\pi}^{-1}(U)$ is a neighborhood of $G$ whenever $U$ is a neighborhood of $\Delta$.

Any neighborhood $U$ of $\Delta$ determines uniquely a covering of $B$ by neighborhoods $N_{U}(b)$ according to the rule $b^{\prime} \in N_{U}(b)$ when $\left(b, b^{\prime}\right) \in U$.

Received by the editors January 13, 1943.

${ }^{1}$ W. Hurewicz and N. E. Steenrod, Proc. Nat. Acad. Sci. U.S.A. vol. 27 (1941) p. 61 .

${ }^{2}$ How well they succeeded in this will be indicated in my next communication. 
However not every covering of $B$ by neighborhoods need arise in this fashion-although the star neighborhoods of any open covering of $B$ may always be so generated.

A slicing function $\phi$ for $\pi$ is any continuous mapping defined over $\bar{\pi}^{-1}(U)$ for some neighborhood $U$ of $\Delta$, with values in $X$, which satisfies the conditions

$$
\begin{aligned}
\pi \phi(x, b) & =b, \\
\phi(x, \pi(x)) & =x,
\end{aligned}
$$

whenever $\phi$ is defined. I shall call $\pi$ a fibre mapping relative to $U$ if it has a slicing function defined over $\bar{\pi}^{-1}(U)$. If $\pi$ is a fibre mapping I shall say that $X$ is a fibre space over the subset $\pi(X)$ of $B$. Since $U$ is a neighborhood of $\Delta, \pi(X)$ is open and closed in $B$.

This new definition is equivalent to the old one if the base space is compact metric (so that the Hurewicz-Steenrod definition is topologically invariant in this case). In fact, for metric space $B$, let $\sigma_{\epsilon}$ denote that neighborhood of $\Delta$ which determines the covering of $B$ by $\epsilon$-spheres. Clearly $X$ is a fibre space (relative to $\pi$ ) over the metric space $\pi(X)$ in the sense of Hurewicz-Steenrod if and only if $\pi$ has a slicing function defined over $\bar{\pi}^{-1}\left(\sigma_{\epsilon}\right)$ for some $\epsilon>0$. Hence, if $\pi$ is $a$ fibre mapping and $\pi(X)$ is compact metrizable then $X$ is a fibre space over $\pi(X)$ in the sense of Hurewicz-Steenrod no matter how $\pi(X)$ is metrized.

Now let $B$ denote an arbitrary metrizable space, let $U$ be a neighborhood of $\Delta$ and let $\pi$ be a fibre mapping whose slicing function is defined over $\bar{\pi}^{-1}(U)$. For simplicity, assume also that $\pi(X)=B$. To show that $X$ is a fibre space in the sense of Hurewicz-Steenrod when $B$ is properly metrized it is clearly sufficient to so metrize $B$ that $\sigma_{\epsilon} \subset U$ for some $\epsilon>0$.

LEMMA. ${ }^{3}$ If $B$ is metrizable and $U$ is an open neighborhood of $\Delta(B)$ then $B$ can be so metrized that $\sigma_{1} \subset U$.

Choose any random metric $d$ for $B$. Since $B \times B$ is metric, hence normal, it is possible to define a continuous function $f \in[0,1]^{B \times B}$ such that

$$
f\left(b, b_{0}\right)=\left\{\begin{array}{lll}
0 & \text { when } & \left(b, b_{0}\right) \in \Delta, \\
1 & \text { when } & \left(b, b_{0}\right) \in B \times B-U .
\end{array}\right.
$$

Let $\phi$ denote the (continuous) mapping $b \rightarrow f_{b}$, where $f_{b}\left(b_{0}\right)=f\left(b, b_{0}\right)$.

3 This proof is modelled after a proof in André Weil, Sur les espaces a structure uniforme et sur la topologie générale, Actualités Scientifiques et Industrielles, no. 551, 1938 , p. 15. 
The graph $B^{\prime}=\sum_{b \in B}(b, \phi(b))$ of $\phi$ is homeomorphic to $B$. The metric of $B^{\prime}$ is induced by the metric of the product $B \times[0,1]^{B}$ and is given by the formula

$$
\delta\left(b_{1}^{\prime}, b_{2}^{\prime}\right)=\left\{d^{2}\left(b_{1}, b_{2}\right)+d^{2}\left(\phi\left(b_{1}\right), \phi\left(b_{2}\right)\right)\right\}^{1 / 2},
$$

where $b$ and $b^{\prime}$ denote corresponding points of $B$ and $B^{\prime}$. If now $\left(b_{1}^{\prime}, b_{2}^{\prime}\right) \in \sigma_{1}$ then $\delta\left(b_{1}^{\prime}, b_{2}^{\prime}\right)<1$, hence $d\left(\phi\left(b_{1}\right), \phi\left(b_{2}\right)\right)<1$, hence $\sup _{b \in B}\left|f\left(b_{1}, b\right)-f\left(b_{2}, b\right)\right|<1$. It follows that $f\left(b_{1}, b_{2}\right)=\mid f\left(b_{1}, b_{2}\right)$ $-f\left(b_{2}, b_{2}\right) \mid<1$, so that $\left(b_{1}, b_{2}\right) \in U$ and $\left(b_{1}^{\prime}, b_{2}^{\prime}\right) \in U^{\prime}$.

THEOREM. If $\pi$ is a fibre mapping and $B$ is metrizable then the metric of $B$ can be so chosen that $X$ is a fibre space over $\pi(X)$ (relative to $\pi$ ) in the sense of Hurewicz and Steenrod.

I conclude by defining uniform homotopy and stating the covering homotopy theorem for general fibre spaces. If $h$ is a homotopy in $B$ of a space $Y$ and $U$ is a neighborhood of $\Delta I$ shall say that $h$ is uniform with respect to $U$ if there is a $\delta>0$ such that $\left|t-t^{\prime}\right|<\delta$ implies that $\sum_{y \in Y}\left(h(y, t), h\left(y, t^{\prime}\right)\right) \subset U$. Let $E_{\delta}=\sum_{0 \leqq t, t^{\prime} \leqq 1,\left|t-t^{\prime}\right|<\delta} \sum_{y \in Y}(h(y, t)$, $\left.h\left(y, t^{\prime}\right)\right)$, so that $E_{0} \subset \Delta$ and $E_{1} \subset B \times B$. Clearly the neighborhoods $U$ with respect to which $h$ is uniform are those which contain an $E_{\delta}$ for some $\delta>0$. Thus $h$ is always uniform with respect to $B \times B$; in the event that $Y$ is compact $h$ is uniform with respect to every neighborhood $U$. I shall call a homotopy $h^{*}$ in $X$ a covering homotopy (with respect to $\pi$ ) if

(1) $\pi h^{*}=h$,

(2) $h_{[0,1]}^{*}(y)$ degenerates to a point whenever $h_{[0,1]}(y)$ degenerates to a point.

I shall refer to the mappings $h_{0}$ and $h_{0}^{*}$ as the initial values of the homotopies $h$ and $h^{*}$, respectively. With these notations the covering homotopy theorem for fibre mappings reads thus.

THEOREM. Given a fibre mapping $\pi \in B^{X}$ relative to $U$, a mapping $g \in X^{Y}$ and a homotopy $h$ in $B$, uniform with respect to $U$, with initial value $\pi g$, there exists a covering homotopy $h^{*}$ in $X$ with initial value $g$.

The covering homotopy $h^{*}$ is constructed stepwise ${ }^{1}$ and is easily seen to be uniform with respect to $U^{*}=\ddot{\pi}^{-1}(U)$ where $\ddot{\pi}\left(x, x^{\prime}\right)$ $=\left(\pi(x), \pi\left(x^{\prime}\right)\right)$. Of course if $U$ is a $\sigma_{\epsilon}$ the neighborhood $U^{*}$ of $\Delta(X)$ need not be a $\sigma_{\epsilon}(X)$.

UNIVERSITY OF ILLINOIS 